$>$ De l'œil de drosophile au muscle de souris, l'histoire des gènes Six est intimement liée à celle des gènes Pax, Eya et Dach. Le premier membre de la famille, cloné chez la drosophile, a été baptisé sine oculis en raison de son rôle dans le développement oculaire, au cours duquel il agit en synergie avec les gènes eyeless (famille Pax), eyes absent (famille Eya) et dachshund (famille Dach). Des résultats récents semblent indiquer que les mécanismes décrits chez la drosophile ont été conservés au cours de l'évolution, pour la différenciation de l'œil de vertébré, comme pour d'autres types de différenciation: Sixl, notamment, semble être un acteur majeur de la myogenèse et participe au développement d'organes tels que les reins, le thymus ou l'oreille interne. Ces différentes fonctions sont à corréler avec la présence de partenaires Pax, Eya et Dach spécifiques dans les nombreux territoires d'expression du gène; elles soulignent également l'importance de ces combinaisons de facteurs pendant l'organogenèse. <

\section{Redéploiement des gènes Six au cours de l'évolution}

Christine Laclef, Pascal Maire

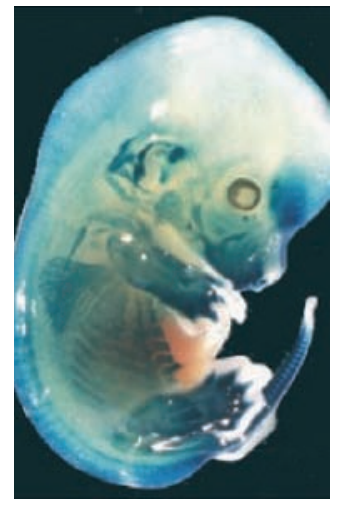

la reconnaissance de séquences spécifiques d'ADN. Le domaine Six participe également à des interactions protéiques avec les cofacteurs Eya (co-activateur) ou Groucho (corépresseur). Ces gènes ont été identifiés chez tous les organismes animaux où ils ont été recherchés, de la planaire à l'homme. On les trouve organisés en clusters (Figure 1 , en bas à droite), et leurs homologies de séquence ont permis de distinguer trois sous-familles chez la drosophile comme chez les mammifères (respectivement 3 et 6 gènes) [1]. La conservation de ces gènes suggère qu'ils participent à des mécanismes essentiels à la survie des organismes.

En outre, des résultats récents indiquent qu'un dérèglement du gène Sixl est associé à certains types de cancers métastatiques (cancer du sein, tumeur de Wilms et rhabdomyosarcome) [2-4]. Ce gène pourrait donc non seulement contrôler la prolifération cellulaire, mais aussi participer à l'acquisition des propriétés invasives des cellules cancéreuses. Les caractéristiques des cellules métastatiques étant remarquablement similaires à celles des cellules embryonnaires, il est probable que l'exploitation des données obtenues au cours du développement devrait permettre de mieux comprendre les mécanismes contrôlant l'engagement des cellules dans un processus cancéreux ou métastatique. 


\section{Caractéristiques fonctionnelles des gènes Six de drosophile}

\section{Sine oculis}

Le premier membre de la famille Six a été isolé chez la drosophile, et baptisé sine oculis (so) car l'absence de ce gène dans les disques imaginaux oculaires entraîne un développement anormal de l'œil $[5,6]$. So participe à l'organogenèse oculaire en collaboration avec les gènes eyeless (ey), eyes absent (eya) et dachshund (dac). Ces quatre gènes interviennent au cours de l'étape précoce de détermination oculaire et peuvent induire, de manière synergique, la formation d'un œil ectopique lorsqu'ils sont surexprimés dans les disques imaginaux de l'antenne ou de la patte [7-9]. Non seulement des boucles de régulation positive relient génétiquement ces quatre gènes, mais leur synergie fonctionnelle semble reposer sur des interactions protéiques directes entre So et Eya, d'une part, et Eya et Dac, d'autre part [10].

\section{Optix}

Un deuxième gène, optix, a été cloné chez la drosophile par homologie de séquence avec le gène Six6/Optix2 de vertébré. II est localisé sur le chromosome 2, à proximité du gène so [11]. Si les profils d'expression de ces deux gènes sont largement superposables dans le disque imaginal de l'œil, leurs caractéristiques fonctionnelles semblent différentes: soit il existe une autre voie d'induction de la différenciation oculaire, parallèle à celle préalablement décrite impliquant ey, so, eya et $d a c$, soit le gène optix agit en aval de cette boucle de régulation génétique.

\section{D-Six4}

Le troisième gène Six de drosophile, D-Six4, est exprimé au cours du développement dans les structures mésodermiques à l'origine des muscles striés squelettiques et de l'enveloppe somatique des gonades [12]. Des mutations ponctuelles de ce gène conduisent à une réduction de la taille des gonades et à des anomalies musculaires: tandis que certains muscles sont absents, d'autres sont présents mais ne s'attachent pas correctement à la cuticule. Enfin, la plupart des cellules musculaires apparaissent mononucléées, ce qui signifie que, chez la drosophile, la perte de fonction de D-Six4 est responsable d'un défaut de fusion des myoblastes en myotubes.

\section{Fonctions des gènes Six chez les vertébrés}

L'étude des profils d'expression des gènes Six au cours du développement embryonnaire chez les vertébrés a permis de proposer un certain nombre d'hypothèses concernant la fonction de ces gènes, hypothèses véri- fiées ensuite par invalidation fonctionnelle ou surexpression du gène d'intérêt.

\section{Six3/Six6}

Les gènes du groupe Six3/Six6 (homologues de optix) sont exprimés très tôt au cours du développement embryonnaire, dans une région de la plaque neurale à l'origine du cerveau antérieur, des placodes optique et nasale. Plusieurs études réalisées chez des vertébrés inférieurs (zebra fish, medaka fish et xénope) ont montré que la surexpression des gènes Six $3 / \operatorname{Six} 6$ peut induire un élargissement des yeux et du cerveau antérieur [13] et, dans certaines conditions, induire la formation de cristallin ou de rétine ectopique [14]. Dans ce dernier cas, la surexpression ectopique de Six 3 induit l'expression de Pax6 là où se forme la rétine additionnelle.

Réciproquement, l'invalidation fonctionnelle de Six 3 chez le medaka fish et chez la souris conduit à l'absence d'œil et de cerveau antérieur, confirmant le rôle majeur joué par Six3 au cours de la différenciation de ces structures chez les vertébrés [15]. En absence de Six3, un certain nombre de facteurs (Wnt1, En2, Otx2, Pax3),

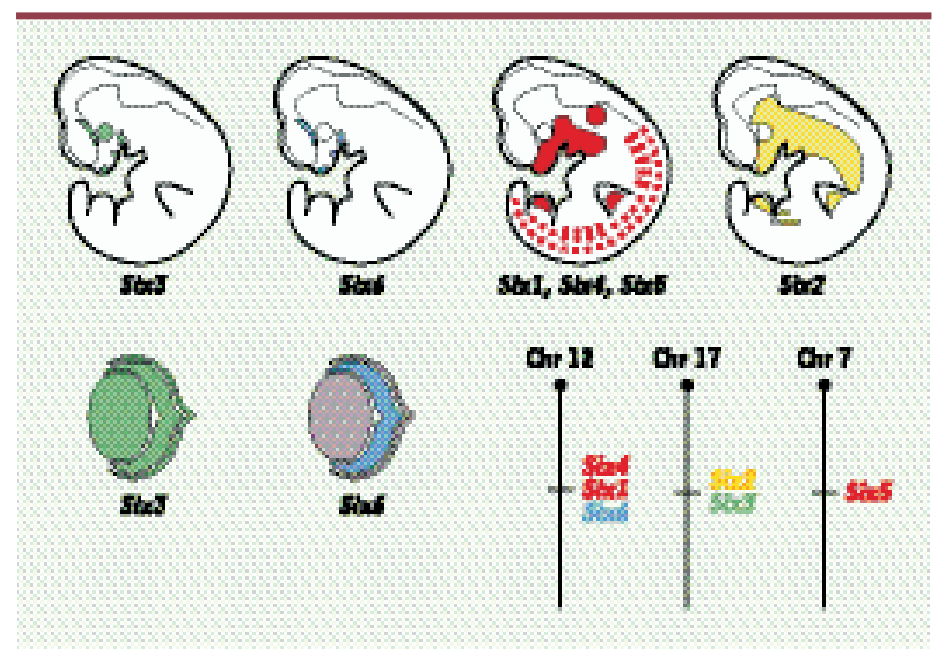

Figure 1. Profils d'expression des 6 gènes Six de souris au cours du développement. Schéma représentant des embryons de souris à 10,5 dpc (day post coitum). À ce stade du développement, l'expression des gènes Six 3 et Six6 est restreinte au cerveau antérieur et à la placode optique. Ces gènes sont ensuite exprimés dans la rétine en cours de différenciation. Six 3 apparaît également exprimé dans la couche pigmentaire et le cristallin. Les gènes Sixl, Six4 et Six 5 sont largement co-exprimés dans les placodes nasales et otiques, dans les arcs branchiaux, dans les ganglions craniaux et dorsaux, ainsi que dans les somites et les bourgeons de membre. Le gène Six2 est, quant à lui, exprimé dans les arcs branchiaux, les bourgeons de membre et le mésenchyme métanéphrique. Par ailleurs, la localisation chromosomique des gènes Six révèle une organisation en cluster de Six4-Six1-Six6 et de Six2-Six3, respectivement sur les chromosomes 12 et 17 . Chr: chromosome (d'après [1]). 
normalement absents, sont exprimés dans le cerveau antérieur. Plus particulièrement, la protéine Six 3 contrôlerait négativement l'expression de Wntl en se liant directement au niveau des séquences régulatrices de ce gène [15].

L'invalidation fonctionnelle de Six6 chez la souris n'est pas létale comme celle de Six3, mais conduit néanmoins à une hypoplasie hypophysaire et à une hypoplasie rétinienne (due à l'absence de nerf et de chiasma optique) [16]. In vitro, il a été montré que Dachl interagit fortement avec Six6 et joue le rôle d'un corépresseur transcriptionnel de Six6 en transfection
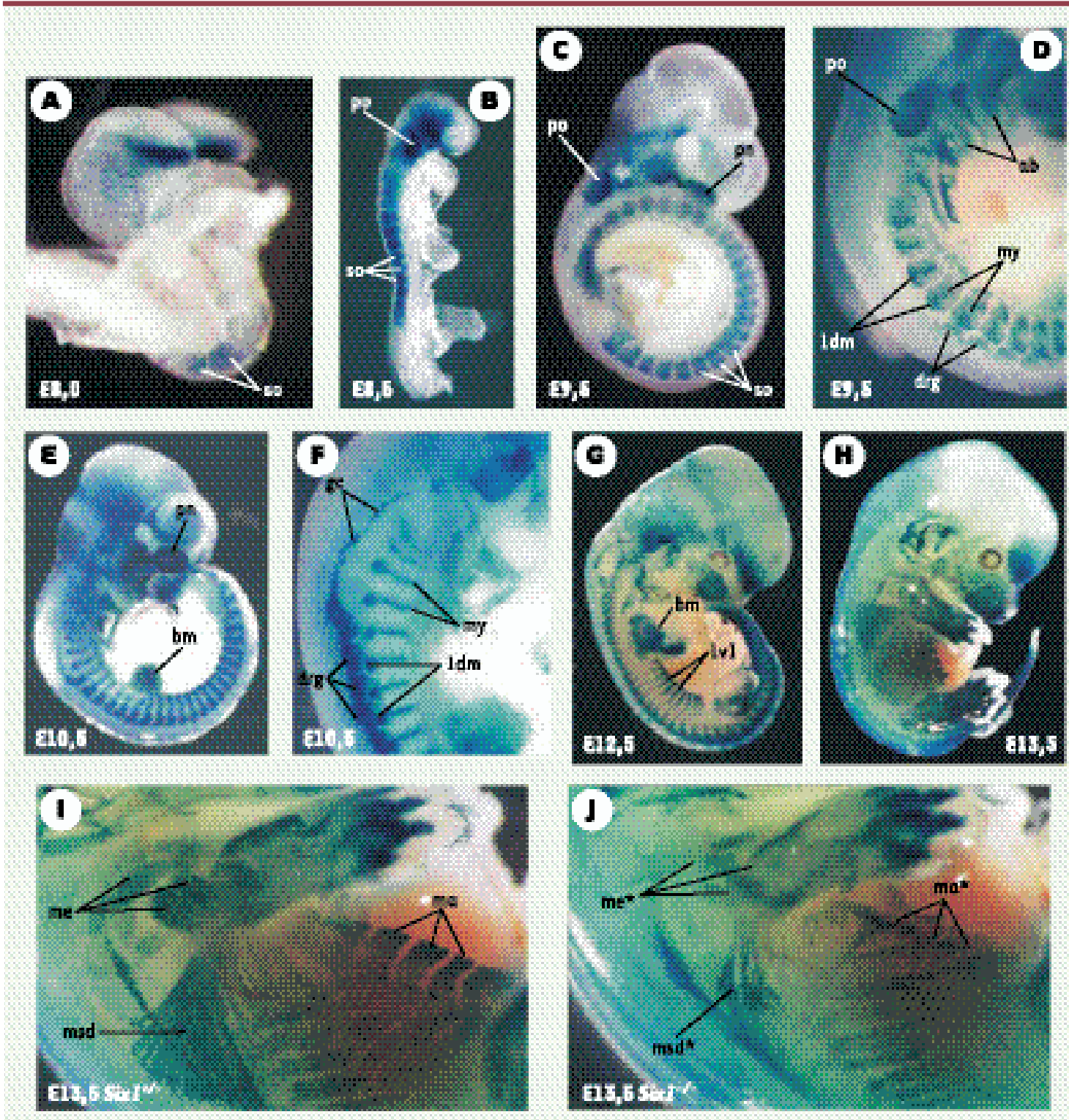

Figure 2. Profil d'expression du gène Sixl au cours du développement et phénotype musculaire des embryons Six ${ }^{-/-}$à $\varepsilon 13,5$ dpc. Le knock-in du gène rapporteur LacZ au locus Sixl permet de visualiser l'expression du gène Sixl par coloration X-gal. $\boldsymbol{A}$ - $\boldsymbol{H}$ : embryons hétérozygotes à différents stades de développement (indiqués en bas à gauche de chaque image). Sixl est exprimé dans de nombreux territoires: les somites (so), la placode otique (po), la placode nasale (pn), les arcs branchiaux (ab), les ganglions dorsaux $(\mathrm{drg})$, les ganglions craniaux proximaux $(\mathrm{gc})$ et les bourgeons de membre $(\mathrm{bm})$. Le marquage somitique apparaît particulièrement fort dans le myotome (my), ainsi que dans les lèvres dorsomédianes (Idm) et ventro-latérales (Ivl) du dermomyotome. I-J: les animaux homozygotes $\operatorname{Six} I^{-/-}(J)$ présentent des défauts musculaires importants à $\varepsilon 13,5$ comparés aux hétérozygotes Six ${ }^{+/-}(\mathrm{I})$. Les muscles abdominaux ( $\mathrm{ma}$ ) et superficiels du dos (msd), notamment, sont réduits et désorganisés, et certains muscles de l'épaule (me) et des membres sont absents. 
transitoire. De plus, le complexe Six6/Dach1 contrôlerait directement l'expression du gène $p 27^{K i p l}$ (codant pour un inhibiteur des kinases dépendantes des cyclines). Les hypoplasies observées apparaissent ici corrélées à un défaut de prolifération des cellules précurseurs [16]. Il est à noter cependant que l'invalidation fonctionnelle de Dachl n'entraîne pas de défauts de prolifération, ni d'hypoplasie hypophysaire chez la souris [17], bien que les nouveau-nés meurent à la naissance pour une raison encore indéterminée [18].

\section{Six $4 /$ Six 5}

Chez les vertébrés supérieurs, les gènes Six 4 et Six 5 sont exprimés dans de nombreux territoires au cours du développement (Figure 1) [19, 20]. L'invalidation fonctionnelle de ces gènes n'entraîne pas de défauts majeurs, mis à part une cataracte développée par les souris dont le gène Six5 est déficient [2022]. Il est probable que ces deux gènes, qui codent pour des protéines fortement homologues et sont exprimés dans les mêmes territoires au cours du développement, aient des fonctions redondantes. L'analyse des souris dont les gènes Six4 et Six 5 ont été invalidés permettra de s'affranchir de cette éventuelle compensation fonctionnelle et de définir plus précisément leurs fonctions.

\section{Six $1 /$ Six2}

Contrairement à leurs homologues de drosophile ou de planaire, les gènes Six 1 et Six2 de vertébrés ne sont pas exprimés dans les territoires oculaires au cours du développement et ne semblent pas particulièrement impliqués dans la différenciation de l'œil [23-25]. En revanche, Sixl est exprimé dans d'autres structures sensorielles, tant au niveau de la tête (placodes nasale et otique, ganglions crâniaux), que dans le reste du corps (ganglions dorsaux, ligne latérale). Chez la souris, le profil d'expression de Sixl est tout à fait comparable à celui de Six4 et de Six5, mais n'est que partiellement superposable à celui de Six2 (Figure 1). Ces profils d'expression suggèrent un redéploiement de l'expression des gènes Sixl/Six2 au cours de l'évolution, et leur participation à de multiples événements de différenciation.

En effet, l'invalidation fonctionnelle du gène Sixl chez la souris a révélé le rôle majeur de ce gène au cours de multiples processus de différenciation. En particulier, Sixl apparaît comme un acteur clé de la différenciation myogénique [26]. Les souris Six $1^{-/-}$meurent à la naissance d'insuffisance respiratoire due à l'absence de diaphragme et à des malformations des côtes. Les nouveau-nés présentent une hypoplasie musculaire qui touche tous les muscles du corps (mais pas les muscles de la tête) et qui atteint plus spécifiquement certains muscles distaux, en particulier au niveau des membres. L'analyse des marqueurs myogéniques exprimés chez ces souris a permis d'établir le rôle tardif de Sixl au cours de la myogenèse primaire (Figure 2). Sixl participe également à l'organogenèse rénale et thymique, à la morphogenèse du squelette craniofacial et thoracique, ainsi qu'à la différenciation de l'oreille interne, de la cavité nasale et des glandes lacrymales et parotides [27-30]. Comme la plupart de ces défauts sont comparables à ceux décrits chez les souris dont le gène Eyal a été invalidé (Figure 3) [31, 32], il est probable que Sixl et Eyal agissent en synergie pour induire le développement de ces organes selon un mécanisme similaire à celui mis en évidence chez la drosophile au cours de l'organogenèse oculaire. Plus généralement, différentes combinaisons de gènes Pax, Six, Eya et Dach pourraient participer à de nombreux processus de différenciation.

Conservation et redéploiement des mécanismes moléculaires impliquant les gènes Pax, Six, Eya et Dach... au cours de la myogenèse

Les gènes Pax3, Six1, Eya2 et Dach2 sont co-exprimés dans les précurseurs myogéniques, au niveau des somites [33]. De plus, une synergie fonctionnelle entre les facteurs Sixl et Eya2, d'une part, et entre Eya2 et Dach2, d'autre part, a été rapportée pour l'induction de la différenciation myogénique chez le poulet [33]. En effet, dans un modèle de somites en culture primaire, la surexpression couplée de ces facteurs induit l'expression de marqueurs de différenciation myogénique tels que MyoD, myogénine et les chaînes lourdes de myosine (MyHC). Le promoteur du gène myogénine possède, en effet, un site MEF3 reconnu par les protéines Six et indispensable à son activité transcriptionnelle au cours du développement de la souris [34]. Par ailleurs, plusieurs combinaisons de facteurs Six et Eya sont capables de se lier au site MEF3 du promoteur myogénine et activent la transcription des gènes rapporteurs avec une efficacité variable $[35,36]$.

Dans l'hypothèse d'une conservation et d'un redéploiement des mécanismes moléculaires impliquant les facteurs Pax, Six, Eya et Dach, l'on s'attend à trouver des phénotypes comparables chez les mutants (Figure 3). De fait, les muscles les plus atteints des souris $S_{i x 1^{-/}}$sont également ceux qui font défaut chez les mutants Splotch (mutants spontanés du gène Pax3) $[26,37]$. II semble donc que Sixl et Pax3 participent à la différenciation des mêmes masses musculaires distales. Néanmoins, Pax3 et Sixl ne contrôlent pas les mêmes étapes de cette voie de différenciation, Pax3 agissant en amont de Sixl. En effet, chez les mutants Splotch, l'absence de muscles hypaxiaux résulte d'un défaut de migration précoce des précurseurs myogéniques [37]. Chez les mutants Sixl, en revanche, la migration n'est pas affectée, mais l'étape ultérieure de différenciation des myoblastes est profondément altérée dans les territoires distaux [26].

\section{... au cours d'autres événements d'organogenèse}

Les mutations des gènes Pax2, Sixl et Eyal conduisent à des défauts d'organogenèse rénale ainsi qu'à des défauts de différenciation de l'oreille interne. Ces phénotypes comparables 
suggèrent qu'une combinaison Six 1-Eyal-Pax2 pourrait participer au développement du rein et de l'oreille (Figure 3) [27].

Des défauts de différenciation thymique et de développement du squelette craniofacial ont été décrits chez les mutants des gènes Paxl, Pax 9 , Sixl et Eyal, suggérant qu'une combinaison Paxl/9-Sixl-Eyal pourrait intervenir au cours du développement de ces structures [27].

Enfin, les mutations du gène Pax6 conduisent au phénotype Small eye, caractérisé par une réduction de la taille des yeux, mais également par des défauts de différenciation de la cavité nasale et des glandes lacrymales et parotides. Ainsi, la combinaison Pax6-Sixl pourrait participer à la différenciation de l'appareil olfactif et des glandes sécrétrices de la face. En revanche, la différenciation oculaire serait contrôlée par une combinaison Pax6-Six3/6 [15].

\section{Conclusions}

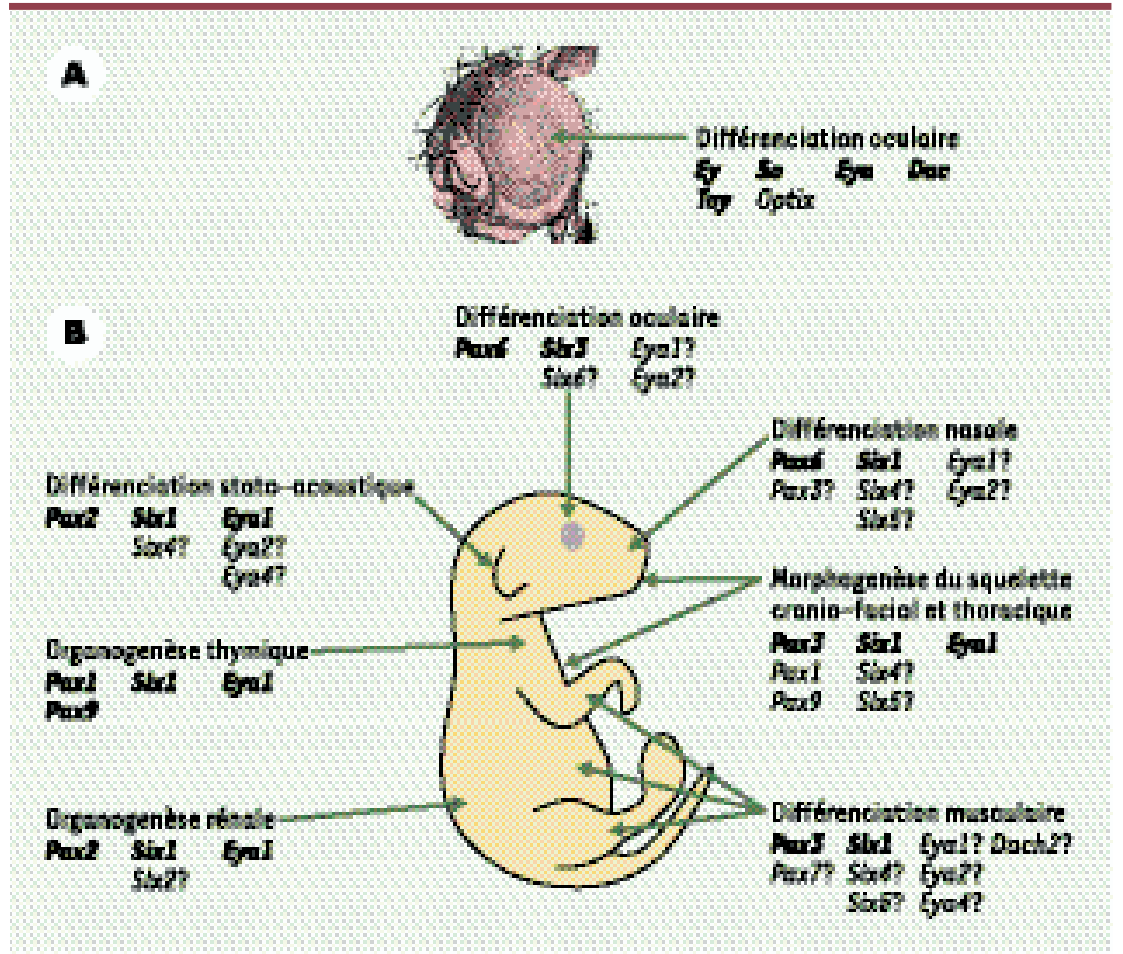

Figure 3. Rôles multiples des gènes Pax, Six, Eya et Dach au cours du développement embryonnaire. $A$. Chez la drosophile, l'organogenèse oculaire est contrôlée par une combinaison de gènes Pax (eyeless, ey et twin of eyeless, toy), Six (sine oculis, so et optix), Eya (eyes absent) et Dach (dachshund, dac). B. Chez la souris, il existe 9 gènes Pax, 6 gènes Six, 4 gènes Eya et 2 gènes Dach. Des combinaisons variables de ces différents facteurs semblent impliquées dans de multiples événements de différenciation, de morphogenèse ou d'organogenèse. Les gènes notés en gras sont ceux pour lesquels l'invalidation fonctionnelle conduit à un développement anormal de l'organe ou du tissus considéré. Les autres gènes Pax, Six, Eya ou Dach, indiqués avec un point d'interrogation, sont exprimés au cours du développement de l'organe en question, mais leur fonction n'a pas encore été clairement démontrée.
L'étude fonctionnelle des homéoprotéines Six a permis de mettre en évidence le rôle d'une nouvelle classe de facteurs de transcription à homéodomaine au cours du développement embryonnaire. Les mécanismes moléculaires impliquant les homéoprotéines Six et leurs partenaires Pax, Eya et Dach apparaissent conservés entre la drosophile et la souris, respectivement pour contrôler l'organogenèse oculaire et la différenciation musculaire. Compte tenu du rôle crucial de ces facteurs au cours de plusieurs événements d'organogenèse, il est vraisemblable que des combinaisons variables de facteurs Pax-Six-Eya-Dach contrôlent de multiples processus de différenciation. Néanmoins, ces corrélations fonctionnelles restent à confirmer par une approche cellulaire et moléculaire, car la plupart des cibles transcriptionnelles de ces facteurs n'ont pas encore été identifiées. $\diamond$

\section{SUMMARY}

Rainbow Six

It has become clear that during evolution, efficient molecular mechanisms are used over and over again to achieve various patterning tasks. The Six gene story illustrates a new aspect of the molecular conservation during embryogenesis. Members of the Six gene family have been identified on the basis of sequence homology with Drosophila sine oculis gene, which acts within a network of genes including eyeless (Pax family), eyes absent (Eya family) and dachshund (Dach family) to trigger compound eye organogenesis. Some aspects of the regulatory complex operating in Drosophila appear to be conserved during vertebrate eye patterning, but also for other differentiation processes. In this regard, Sixl is required nonetheless during myogenesis, but also for kidney, thymus, inner ear, nose, lacrimal and salivary gland organogenesis. These phenotypes are reminiscent of those previously described for Eya and Pax mutants, suggesting a functional link between these factors during mammalian organogenesis. $\diamond$ 


\section{RÉFÉRENCES}

1. Kawakami K, Sato S, Ozaki H, Ikeda K. Six family genes. Sructure and function as transcription factors and their roles in development. Bioessays $2000 ; 22: 616-26$.

2. Ford HL, Kabingu EN, Bump $\varepsilon A$, et al. Abrogation of the $G 2$ cell cycle checkpoint associated with overexpression of HSIXI: a possible mechanism of breast carcinogenesis. Proc Natl Acad Sci USA 1998; 95: 12608-13.

3. Li CM, Guo M, Borczuk A, et al. Gene expression in Wilms' tumor mimics the earliest committed stage in the metanephric mesenchymal-epithelial transition. Am J Pathol 2002; 160: 2181-90.

4. Yu Y, Khan J, Khanna C, et al. Expression profiling identifies the cytoskeletal organizer ezrin and the developmental homeoprotein Six-1 as key metastatic regulators. Nat Med 2004; $4: 4$.

5. Cheyette BN, Green PJ, Martin K, et al. The Drosophila sine oculis locus encodes a homeodomain-containing protein required for the development of the entire visual system. Neuron 1994; 12: 977-96.

6. Serikaku MA, O'Tousa JE. Sine oculis is a homeobox gene required for Drosophila visual system development. Genetics 1994; 138: 1137-50.

7. Halder $G$, Callaerts $P$, Flister $S$, et al. Eyeless initiates the expression of both sine oculis and eyes absent during Drosophila compound eye development. Development 1998; 125: 2181-91.

8. Bonini NM, Bui QT, Gray-Board GL, Warrick JM. The Drosophila eyes absent gene directs ectopic eye formation in a pathway conserved between flies and vertebrates. Development 1997; 124: 4819-26.

9. Shen W, Mardon G. Ectopic eye development in Drosophila induced by directed dachshund expression. Development 1997; 124: 45-52.

10. Pignoni F, Hu B, Zavitz KH, et al. The eye-specification proteins So and Eya form a complex and regulate multiple steps in Drosophila eye development. Cell 1997; 91 : 881-91.

11. Seimiya M, Gehring WJ. The Drosophila homeobox gene optix is capable of inducing ectopic eyes by an eyeless-independent mechanism. Development 2000; 127 : 1879 86.

12. Kirby RJ, Hamilton GM, Finnegan DJ, et al. Drosophila homolog of the myotonic dystrophy-associated gene, $S I X 5$, is required for muscle and gonad development. Curr Biol 2001; 11: 1044-9.

13. Kobayashi M, Toyama R, Takeda H, et al. Overexpression of the forebrain-specific homeobox gene six 3 induces rostral forebrain enlargement in zebrafish. Development 1998; 125: 2973-82

14. Loosli F, Winkler S, Wittbrodt J. Six3 overexpression initiates the formation of ectopic retina. Genes Dev 1999; 13: 649-54.

15. Lagutin 0 , Zhu C, Kobayashi D, et al. Six3 repression of Wnt signaling in the anterior neuroectoderm is essential for vertebrate forebrain development. Genes Dev 2003; 17: 368-79.

16. Li X, Perissi V, Liu F, et al. Tissue-specific regulation of retinal and pituitary precursor cell proliferation. Science 2002; 297: 1180-3.

17. Backman M, Machon 0, Van Den Bout CJ, Krauss S. Targeted disruption of mouse Dachl results in postnatal lethality. Dev Dyn 2003; 226: 139-44.

18. Davis RJ, Shen W, Sandler Yl, et al. Dachl mutant mice bear no gross abnormalities in eye, limb, and brain development and exhibit postnatal lethality. Mol Cell Biol $2001 ; 21: 1484-90$
19. Esteve P, Bovolenta P. cSix4, a member of the six gene family of transcription factors, is expressed during placode and somite development. Mech Dev 1999; 85: 161-5.

20. Ozaki $\mathrm{H}$, Watanabe $\mathrm{Y}$, Takahashi $\mathrm{K}$, et al. Six4, a putative myogenin gene regulator, is not essential for mouse embryonal development. Mol Cell Biol 2001; 21: 3343-50.

21. Klesert TR, Cho DH, Clark Jl, et al. Mice deficient in Six 5 develop cataracts: implications for myotonic dystrophy. Nat Genet 2000; 25 : 105-9.

22. Sarkar PS, Appukuttan B, Han J, et al. Heterozygous loss of Six 5 in mice is sufficient to cause ocular cataracts. Nat Genet $2000 ; 25: 110-4$.

23. Oliver $\mathrm{G}$, Wehr $\mathrm{R}$, Jenkins $\mathrm{N}$, et al. Homeobox genes and connective tissue patterning. Development 1995; 121: 693-705.

24. Pandur PD, Moody SA. Xenopus Sixl gene is expressed in neurogenic cranial placodes and maintained in the differentiating lateral lines. Mech Dev 2000; 96 : $253-7$.

25. Ghanbari H, Seo HC, Fjose A, Brandli AW. Molecular cloning and embryonic expression of Xenopus Six homeobox genes. Mech Dev 2001; 101: 271-7.

26. Laclef C, Hamard G, Demignon J, et al. Altered myogenesis in Sixl-deficient mice. Development 2003; 130: 2239-52.

27. Laclef C, Souil $\varepsilon$, Demignon J, Maire P. Thymus, kidney and craniofacial abnormalities in Six 1 deficient mice. Mech Dev 2003; 120: 669-79.

28. Xu PX, Zheng W, Huang L, et al. Sixl is required for the early organogenesis of mammalian kidney. Development 2003; 130 : 3085-94.

29. Zheng $W$, Huang L, Wei ZB, et al. The role of Sixl in mammalian auditory system development. Development 2003; 130 : 3989-4000.

30. Ozaki H, Nakamura K, Funahashi J, et al. Sixl controls patterning of the mouse otic vesicle. Development 2004; 131: 551-62.

31. Xu PX, Adams J, Peters H, et al. Eyal-deficient mice lack ears and kidneys and show abnormal apoptosis of organ primordia. Nat Genet 1999; 23: 113-7.

32. Xu PX, Zheng $W$, Laclef $C$, et al. Eyal is required for the morphogenesis of mammalian thymus, parathyroid and thyroid. Development 2002; 129: 3033-44.

33. Heanue TA, Reshef R, Davis RJ, et al. Synergistic regulation of vertebrate muscle development by Dach2, Eya2, and Six1, homologs of genes required for Drosophila eye formation. Genes Dev 1999; 13: 3231-43.

34. Spitz F, Demignon J, Porteu A, et al. Expression of myogenin during embryogenesis is controlled by Six/sine oculis homeoproteins through a conserved MEF3 binding site. Proc Natl Acad Sci USA 1998; 95 : 14220-5.

35. Ohto H, Kamada S, Tago K, et al. Cooperation of six and eya in activation of their target genes through nuclear translocation of Eya. Mol Cell Biol 1999; 19: 6815-24.

36. Fan X, Brass LF, Poncz M, et al. The alpha subunits of $\mathrm{Gz}$ and $\mathrm{Gi}$ interact with the eyes absent transcription cofactor Eya2, preventing its interaction with the six class of homeodomain-containing proteins. J Biol Chem 2000; 275 : 32129-34.

37. Goulding M, Sterrer S, Fleming J, et al. Analysis of the Pax-3 gene in the mouse mutant splotch. Genomics 1993; 17: 355-63.
TIRÉS À PART

C. Laclef 